УДК 537.9

\title{
Analysis of Superconductor Magnetization Hysteresis
}

\author{
Denis M. Gokhfeld* \\ Kirensky Institute of Physics \\ Federal Research Center KSC SB RAS \\ Akademgorodok, 50/38, Krasnoyarsk, 660036 \\ Russia
}

Received 05.12.2016, received in revised form 10.12.2017, accepted 20.03.2018

The critical state model and the extended critical state model are described to analyse a magnetization hysteresis and to find superconductor parameters. We discuss how geometric sizes and form influence on magnetization hysteresis, critical current and trapped flux.

Keywords: pinning, Abrikosov vortices, critical state, critical current density, trapped flux. DOI: $10.17516 / 1997-1397-2018-11-2-219-221$.

An essential criterion of superconductivity in studied samples is sharp drop of the resistance at some temperature. Other essential criterion is a great diamagnetic response during the magnetization measurements. Temperature and magnetic field dependencies of the magnetization give the detailed characterization of the superconducting phase in single-crystal and polycrystalline samples.

The critical state model [1] and its many modifications describe entering and trapping of magnetic flux in a II-type superconductor. The extended critical state model [2-4] accounts contributions to the magnetization loop from the surface and the internal volume of a superconducting sample. The extended model allows to parametrize the magnetization hysteresis with asymmetry relative to the $M=0$ axis.

\section{Determination of superconducting parameters from magnetic measurements}

The critical current density $j_{c}$ is most important parameter for superconductor applications. The diamagnetic response is determined by $j_{c}$. The critical state model [1] results in the Bean formula $j_{c}=3 \Delta M / 2 R$, such that one can estimate $j_{c}$ from the magnetic measurement. In the Bean formula $\Delta M$ is the hysteresis width, $R$ is the radius of the current circulation.

Main stages of magnetization hysteresis analysis are given below.

1. The content of a superconducting phase should be estimated firstly. The virgin magnetization changes as $M(H)=-x H$ for $H<H_{c 1}$, here $x$ is the content of a superconducting phase. The demagnetization factor can distort the value of $x$. Additional diamagnetic [5] / paramagnetic $[6,7]$ phases in a sample tilt the magnetization hysteresis clockwise / anticlockwise. Coexistence of superconducting and ferromagnetic phases in a sample gives a composite hysteresis [8]. For hysteresis of the superconducting phase the dependence of $M$ on $H$ approaches to 0 as $H$ increases to the upper critical field $H_{c 2}$.

2. Some special fields are remarkable on a hysteresis and can be estimated directly (Fig. 1). The lower critical field $H_{c 1}$ is the point in which the virgin $M(H)$ dependence begins to deflect from the linear line. The virgin $M(H)$ dependence becomes to coincide with the envelop magnetization loop at $H=H_{p}$, the full penetration field. The $M(H)$ dependence becomes reversible at $H$ higher than the irreversibility field $H_{i r r}$. The superconductivity and the corresponding

*gokhfeld@iph.krasn.ru

(c) Siberian Federal University. All rights reserved 
diamagnetic response disappear at $H$ higher than the upper critical field $H_{c 2}$. The trapped magnetic flux $B_{t r}$ in a sample equals to $\mu_{0} \Delta M$ at $H=0$.

3. The depth of the magnetic field penetration $\lambda_{0}$ can be estimated from the reversible part of magnetization hysteresis. The London model [9] results in formula for the equilibrium magnetization $M=-\phi_{0} /\left(32 \pi^{2} \lambda_{0}^{2}\right) \ln \left(\eta H_{c 2} / H\right)$, where $\phi_{0}$ is the magnetic flux quantum, $\eta$ is a constant about 1.

4. The hysteresis width is defined by the product of $j_{c}$ and $R$ such that the current circulation scale should be found for estimations of $j_{c}$ from magnetic measurements. There is a problem here because the size of current circulation $R$ can differ from the sample radius. It may be the effective grain radius for polycrystalline samples or a size of grain clusters. The hysteresis asymmetry relative to the $M=0$ axis allows to estimate $R$ because this asymmetry depends on $R$ but it is independent of $j_{c}$. The asymmetry is defined by the ratio of $l_{s} / R$, where $l_{s}$ is the depth of the surface layer with equilibrium magnetization [2,3]. Abrikosov vortices are not pinned in this surface layer. The value of $l_{s}$ is not larger than $\lambda_{0}$. Noticeable asymmetry of hysteresis is observed for $l_{s} / R>0.1$ such that one can estimate $R<10 \lambda$ for asymmetric magnetization hysteresis. For analyzed polycrystalline superconductors the magnetization loops are good fitted by the extended critical state model with $R$ equal to the average grain radius obtained from SEM.

5. The $j_{c}(H)$ dependencies are easy plotted from the magnetization loops by using the Bean formula. These $j_{c}$ values obtained are the critical current density averaged on the cross-section perpendicular to the external magnetic field $H$. Then the field dependence of the pinning force $F_{p}(H)=\mu_{0} H j_{c}(H)$ is plotted that allows to find the maximal pinning field and $H_{\text {irr }}$ also. If the second peak (fishtail, peak effect) is noticeable on a magnetization hysteresis and on field dependencies of $j_{c}$ and $F_{p}$ than the peak position and its temperature evolution contain information about the vortex lattice transition or the phase separation in superconductor [5].

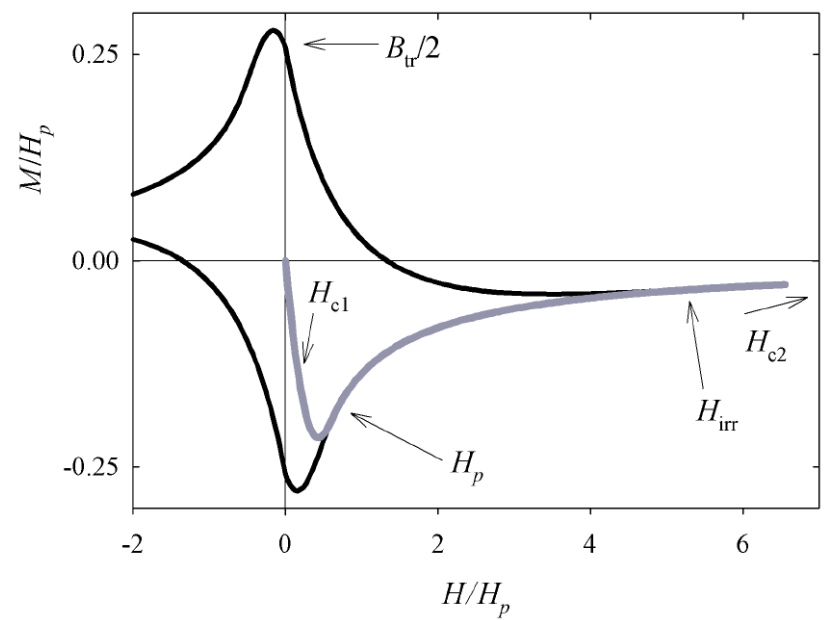

Fig. 1. Characteristic fields on magnetization hysteresis of superconductor. The $H_{c 2}$ field is more larger than a maximal external field

As the extended critical state model describes, the averaged critical current density $j_{c}$ depends on the size and the form of the sample. Due to the surface layer the averaged critical current $j_{c}=j_{c b}\left(1-l_{s}(H) / R\right)^{n}$, here $j_{c b}$ is the critical current density of a macroscopic sample with sizes more larger than $\lambda_{0}, n$ is the parameter defined by the sample form, $n=3$ for the long cylindric sample and $n=2$ for the long plate parallel to $H$ (Fig. 1).

Review article [3] has some additional references on application of the extended critical state model to analysis of magnetization loops of different superconductors. 


\section{Conclusion}

Magnetization hysteresis analysis gives some parameters of superconductors: the lower $H_{c 1}$ and upper $H_{c 2}$ critical fields, the full penetration field $H_{p}$, the irreversibility field $H_{i r r}$, the trapped flux $B_{t r}$, the equilibrium layer depth $l_{s}$, the critical current density $j_{c}$. When sizes of a sample/grains are comparable with the magnetic field penetration depth $\lambda_{0}$, the magnetization hysteresis is asymmetric and the extended critical state model should be applied to describe this. The averaged critical current density decreases with the sample/grain sizes due to equilibrium magnetization of the surface layer. Also the sample/grain sizes and their form influence on the magnetization hysteresis form, particularly on the pinning force maximum field.

The work is supported by Russian Foundation for Basic Research (grant 16-42-240445).

\section{References}

[1] C.P.Bean, Magnetization of hard superconductors, Phys. Rev. Lett., 8(1962), 250.

[2] D.M.Gokhfeld et al., Magnetization asymmetry of type-II superconductors in high magnetic fields, J. Appl. Phys., 109(2011), 033904.

[3] D.M.Gokhfeld, An extended critical state model: Asymmetric magnetization loops and field dependence of the critical current of superconductors, Phys. Solid State, 56 (2014), 2298.

[4] D.M.Gokhfeld, Critical current density and trapped field in HTS with asymmetric magnetization loops, J. Phys.: Conference Series, 695(2016), 012008.

[5] D.A.Balaev et al., Increase in the Magnetization Loop Width in the $\mathrm{Ba}_{0.6} \mathrm{~K}_{0.4} \mathrm{BiO}_{3}$ Superconductor: Possible Manifestation of Phase Separation, J. Exp. Theor. Phys., 118(2014), 104.

[6] E.Altin, D.M.Gokhfeld, F.Kurt, M.E.Yakinci, Physical, electrical, transport and magnetic properties of $\mathrm{Nd}(\mathrm{Ba}, \mathrm{Nd})_{2.1} \mathrm{Cu}_{3} \mathrm{O}_{7}$ system, J. Mater. Sci.: Mater. Electron., 24(2013), 5075.

[7] E.Altin et al., Vortex pinning and magnetic peak effect in $\mathrm{Eu}(\mathrm{Eu}, \mathrm{Ba})_{2.125} \mathrm{Cu}_{3} \mathrm{O}_{x}, J$. Mater. Sci.: Mater. Electron., 25(2014), 1466.

[8] E.Altin et al., Hysteresis loops of $\mathrm{MgB}_{2}+$ Co composite tapes, J. Mater. Sci.: Mater. Electron., 24(2013), 1341.

[9] Z.Hao, J.R.Clem, Phys. Rev. Lett., 67(1991), 2371.

\section{Анализ петель намагниченности сверхпроводников}

Денис М. Гохфельд

Институт физики им. Л. В. Киренского СО РАН

Академгородок, 50/38, Красноярск, 660036

Россия

\footnotetext{
Описано использование модели критического состояния и расширенной модели критического состояния для определения параметров сверхпроводников из измеренных петель намагниченности. Обсуждается влияние геометрических размеров и формы образцов на вид петель намагниченности, критический ток и замороженное магнитное поле.
}

Ключевые слова: пиннинг, вихри Абрикосова, критическое состояние, плотность критического тока, захваченное поле. 